\title{
Co-rotational Formulation for Bonded Joint Finite Elements
}

\author{
Scott E. Stapleton ${ }^{1}$ and Anthony M. Waas ${ }^{2}$ \\ University of Michigan, Ann Arbor, MI, 48109, USA \\ and \\ Steven M. Arnold ${ }^{3}$ and Brett A. Bednarcyk ${ }^{4}$ \\ NASA Glenn Research Center, Cleveland, OH, 44135, USA
}

\begin{abstract}
Enhanced finite elements are elements with an embedded analytical solution that can capture detailed local fields, enabling more efficient mesh independent finite element analysis. In earlier research, this method was applied to adhesively bonded joints. The adherends were modeled as composite Euler-Bernoulli beams, and the adhesive layer was modeled as a bed of linear shear and normal springs. The field equations were derived using the principle of minimum potential energy, and the resulting solutions for the displacement fields were used to generate shape functions and a stiffness matrix for a single bonded joint finite element. In this study, the capability to model large rotations and non-linear adhesive constitutive behavior is developed, and progressive failure of the adhesive is modeled by remeshing the joint as the adhesive fails. The results obtained using this enhanced joint element is compared with experimental results.
\end{abstract}

\section{Introduction}

$\mathrm{W}$ ITH the increased use of fiber reinforced composite materials, adhesively bonded joints become an increasingly critical topic. As bonded joints increase in popularity and use, the demand for modeling techniques increases also. In the past, analytical models have been favored as the preferred method of predicting stresses and strength ${ }^{1-5}$, but finite element (FE) methods have emerged as the new standard in preliminary design due to necessity of analyzing and designing components that contain multiple joints where analytical techniques become intractable. FE based methods have been proven to be extremely powerful, but the small scale of the adhesive thickness when compared to the dimensions of the surrounding structure has kept joint FE analysis largely out of global vehicle models. A fine mesh is needed to correctly model the adhesive layer producing an incompatibility in simultaneously analyzing the joint stresses accurately in conjunction with a very coarse model of an entire vehicle. Therefore, the actual design and sizing of joints is often put off until a later time, when small submodels are used to look into the details of a vehicle.

To address this problem, a bonded joint finite element has been created ${ }^{6-9}$. This joint element considers the adherends to behave like beams and the adhesive to be made up of a bed of shear on normal springs. The governing equations of this structural model are found and solved to produce enhanced shape functions. Furthermore, the element has been generalized to allow multiple adherend/adhesive layers and ply drops/thickness tapers, providing the capability to model various different joint types with very few elements.

This paper presents and extension of the joint element to model progressive failure of a joint and ultimately predict the strength using very few elements. Modern polymeric adhesives are usually highly nonlinear, causing linear elastic analysis to be insufficient. Furthermore, the eccentricity of many joint configurations results in large rotations early on in the loading ${ }^{5,10,11}$, necessitating the consideration of nonlinear geometric effects

Therefore, geometric nonlinear effects due to large rotations and material nonlinearity are pivotal in predicting the strength of a joint. This paper will extend the previously created joint element to include these effects.

\footnotetext{
${ }^{1}$ Graduate Student, Department of Aerospace Engineering. AIAA Student Member. sstaple@umich.edu

${ }^{2}$ Professor, Department of Aerospace Engineering. AIAA Associate Fellow. dcw@umich.edu.

${ }^{3}$ Chief, Mechanics and Life Prediction, 21000 Brookpark Rd. AIAA Member. Steven.M.Arnold@NASA.gov

${ }^{4}$ Material Research Engineer, 21000 Brookpark Rd. AIAA Senior Member. Brett.A.Bednarcyk@NASA.gov
} 
Additionally, a method of growing an adhesive crack internally within an element during the analysis will be presented in order to preserve the original intent of the joint element, which is to model a joint with very few elements.

Currently, the scientific community seems to model the progressive failure of joints with dense-mesh finite elements using damage mechanics methods like cohesive zone models, or continuum mechanics ${ }^{12}$. Since the joint element is merely a tool, it will accommodate using inputs derived from either of these philosophies to govern the stress-strain relation of the adhesive. A method will be shown of characterizing the adhesive layer using either bulk adhesive tensile data as would someone using continuum mechanics damage progression, or fracture mechanics inputs like mode I strength and fracture toughness. The application of each will be demonstrated and results will be compared with published experiments.

\section{Formulation}

The formulation of the joint element has been broken up into discrete parts, namely the co-rotational formulation, material nonlinearities, crack growth, and adhesive constitutive modeling. Each section presents a formulation to address a certain aspect of the progressive failure of the joints. The co-rotational formulation addresses large rotations in joint problems while material nonlinearities show how nonlinear constituents are modeled. The crack growth formulation deals with the failure of the adhesive layer. Finally, the last section illustrates a few methods of defining the properties of the adhesive based on several different experimental techniques.

\section{A. Co-Rotational Formulation}

Consider a structure consisting of $N$ layers of thin plates under cylindrical bending joined together by $N-1$ thin layers of a much more compliant adhesive material (see Figure 1a). The plates are assumed to behave as "wide" Euler Bernoulli beams (hence the cylindrical bending assumption). The adhesive joining the plates is modeled as a Winkler foundation. The plates can be isotropic, transversely isotropic, or a layered composite. The plates and adhesive are assumed to be under proportional loading, and are modelled as nonlinear elastic materials.
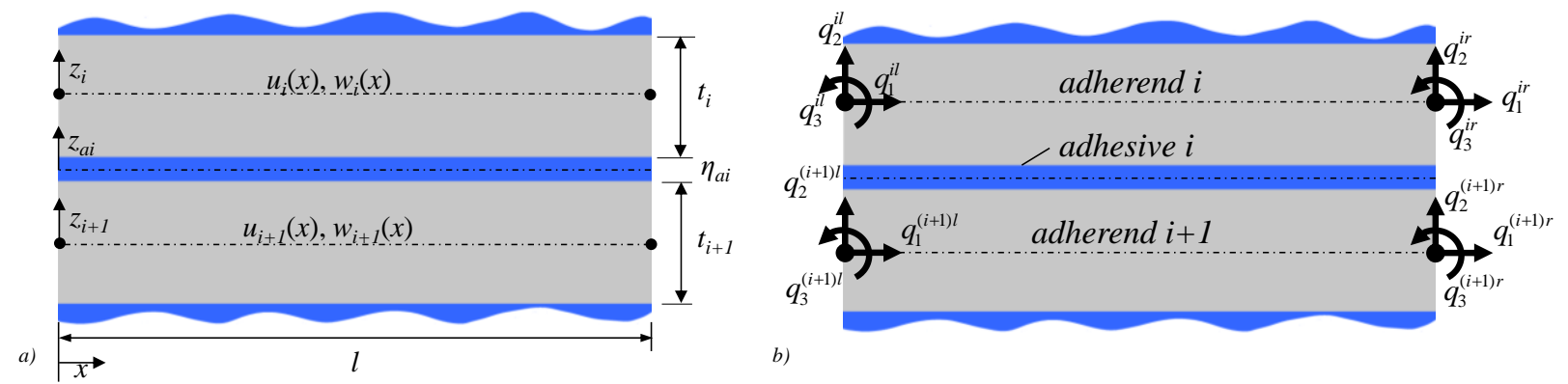

Figure 1. Overlap region of an adhesively bonded joint with multiple bonded layers: a) geometric parametrs (width in the $y$-direction is $b$ ) and b) finite element discretization.

A co-rotational formulation is used to capture large rotations, and has been primarily adapted from prior work by Belutschko and Hsieh ${ }^{13}$ and Crisfield and Moita ${ }^{14}$. This formulation tracks the rigid body rotation of an element through a local rotational coordinate system, and considers the rotations and deformations measured with respect to this rotated frame of reference to be small. The main benefit of this formulation is that the previously implemented code for the small rotation problem ${ }^{7-9}$ can be utilized in subsequent calculations.

The element has $2 N$ nodes located at the boundaries of the centerline of the plates (numbered as shown in Figure $1 b)$, and the nodal displacements are defined as:

$$
\mathbf{q}=\left[\begin{array}{lllll}
\mathbf{q}^{1} & \ldots & \mathbf{q}^{i} & \ldots & \mathbf{q}^{N}
\end{array}\right]^{T}
$$

where the superscript represents the adherend or plate, and 


$$
\mathbf{q}^{i}=\left[\begin{array}{llllll}
q_{1}^{i l} & q_{2}^{i l} & q_{3}^{i l} & q_{1}^{i r} & q_{2}^{i r} & q_{3}^{i r}
\end{array}\right]
$$

refers to the horizontal, vertical, and rotational displacements of the left and right nodes in plate $i$ respectively.

\section{Rigid Body Displacements}

The element has a local rotated coordinate system, $\hat{\mathbf{x}}$, which is rotated and translated relative to the fixed coordinate system, $\mathbf{x}$, by an angle $\phi$ and a vector $\mathbf{q}_{t}^{1}$ respectively (Figure 2). The translation and rotation will be properly defined later. The nodal displacements of the element in the fixed coordinate system can be decomposed into rigid body displacements, $\mathbf{q}_{r i g}$, and displacements which only cause deformation in the body, $\mathbf{q}_{d e f}$, with the relation:

$$
\mathbf{q}=\mathbf{q}_{r i g}+\mathbf{q}_{d e f}
$$

The rigid body nodal displacements, $\mathbf{q}_{r i g}$, can be further decomposed into rigid body displacements resulting from rigid body rotation, $\mathbf{q}_{r}$, and displacements resulting from rigid body translation, $\mathbf{q}_{t}$ :

$$
\mathbf{q}_{\text {rig }}=\mathbf{q}_{t}+\mathbf{q}_{r} .
$$
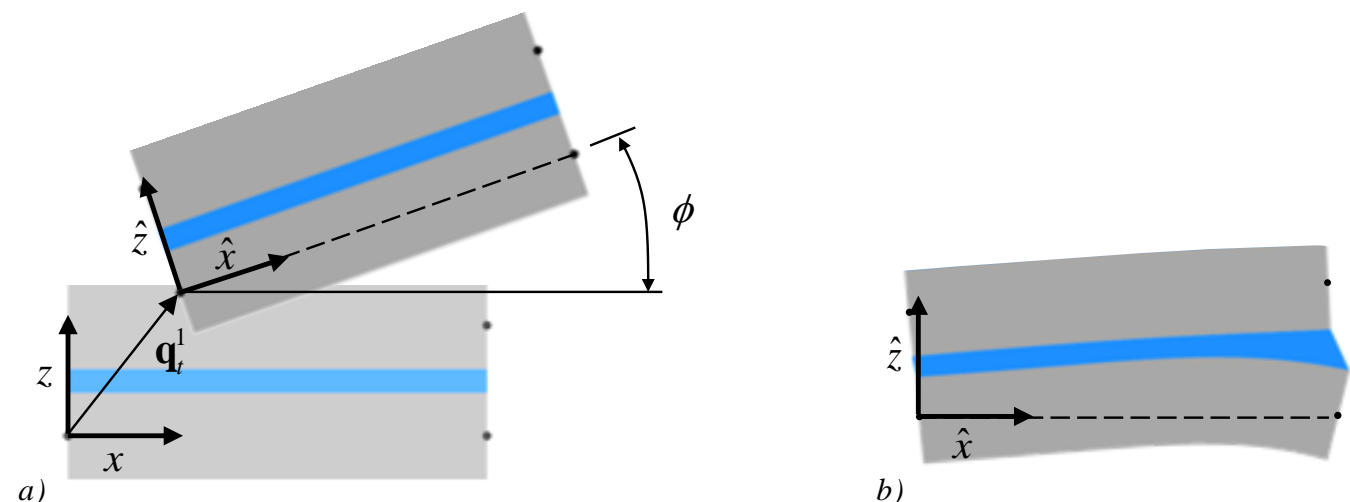

b)

Figure 2. The nodal displacements can be broken up into two parts: a) rigid translation and rotations and b) local deformations.

The translational displacements, $\mathbf{q}_{t}$, are defined as:

$$
\mathbf{q}_{t}=\left[\begin{array}{lll}
\mathbf{q}_{t}^{1} & \ldots & \mathbf{q}_{t}^{1}
\end{array}\right]^{T}
$$

which is the horizontal and vertical displacements of the left node of the first plate and the rotation of the first adherend:

$$
\mathbf{q}_{t}^{1}=\left[\begin{array}{lll}
q_{1}^{1} & q_{2}^{1} & \phi
\end{array}\right]
$$

Although the rotation is not necessarily part of the rigid body translation, it is more convenient to insert it into the translational rigid body displacements because each adherend will be rigidly rotated by the angle $\phi$.

To find the rigid body displacements due to the rotation of the element about the first node, consider the right node of the $i$ th adherend, node ir (Figure 3a). Initially, node ir can be located relative to the first node by a position vector $\mathbf{x}_{i r}$. When the element rotates about the first node by the angle $\phi$, its new position relative to the first node can be expressed by an orthogonal transformation matrix as $\mathbf{T}_{1}^{T} \mathbf{x}_{i r}$, where 


$$
\mathbf{T}_{1}=\left[\begin{array}{ccc}
c & s & 0 \\
-s & c & 0 \\
0 & 0 & 1
\end{array}\right]
$$

and $s$ and $c$ denote the sine and cosine of the angle $\phi$. Therefore, the displacement vector, $\mathbf{q}_{r o t}^{i r}$, of node $i r$ due to rigid body rotation can be expressed as

$$
\mathbf{q}_{r o t}^{i r}=\left(\mathbf{T}_{1}^{T}-\mathbf{I}\right) \mathbf{x}_{i r}
$$
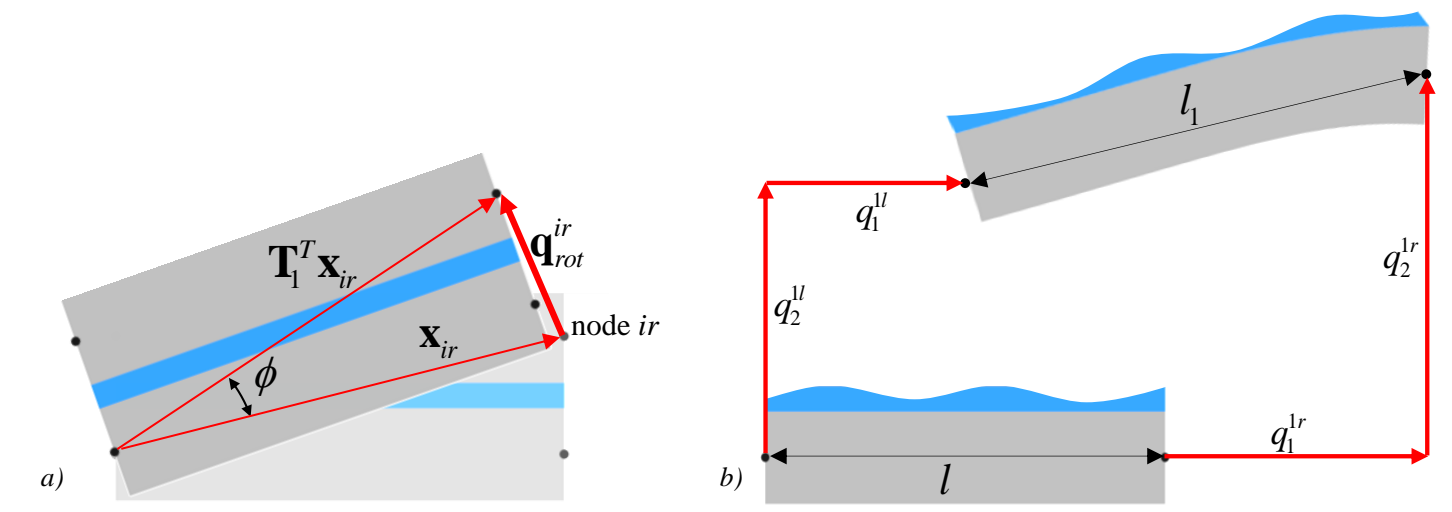

Figure 3. Displacements a) $\mathbf{q}_{r o t}^{i r}$ of node ir due to a rigid body rotation of the joint element $b$ ) and initial and current lengths of the 1st adherend are used to determine the rotation angle..

Translating this to all nodes and combining with Equation 4, the displacements due to rigid body rotation are

$$
\mathbf{q}_{r i g}=\mathbf{q}_{t}+\left(\mathbf{T}^{T}-\mathbf{I}\right) \mathbf{X}
$$

where

$$
\mathbf{T}=\left[\begin{array}{lll}
\mathbf{T}_{1} & & \\
& \ddots & \\
& & \mathbf{T}_{1}
\end{array}\right]
$$

and the vector $\mathbf{X}$ is simply a collection of the initial $x$ and $z$ coordiantes of the nodes, and is defined explicitly as

$$
\mathbf{X}=\left[\begin{array}{llllllll}
\mathbf{X}_{1 l} & \mathbf{X}_{1 r} & \ldots & \mathbf{X}_{i l} & \mathbf{X}_{i r} & \ldots & \mathbf{X}_{N l} & \mathbf{X}_{N r}
\end{array}\right]^{T}
$$

where the first subscript identifies the plate number, and the following letter, either $l$ or $r$, refers to the left or right node respectively. The nodal coordinate vector for the $i$ th adherend and the left node is defined as

$$
\mathbf{X}_{i l}=\left[\begin{array}{lll}
x_{i l} & z_{i l} & 0
\end{array}\right]
$$

while the coordinate vector of the right node is defined in an identical fashion.

2. Determination of the Rotation Angle

If the rotation is not constant within the joint, the rotation angle is an approximation. Adhering to the conventional approach for co-rotational beam formulations, the rotation angle was chosen to be the rotation of the 
first adherend as shown in Figure 3a. To find the transformation matrix of Equation 7, the sine and cosine of the rotation angle can be expressed as

$$
s=\sin \phi=\frac{l_{1 z}}{l_{1}}
$$

and

$$
c=\cos \phi=\frac{l_{1 x}}{l_{1}}
$$

which are defined in terms of the nodal displacements of the first adherend by

$$
\begin{gathered}
l_{1 x}=l+q_{1}^{1 r}-q_{1}^{1 l}, \\
l_{1 z}=q_{2}^{1 l}-q_{2}^{1 r}
\end{gathered}
$$

where $l$ is the original length of the element and $l_{l}, l_{1 x}$, and $l_{1 z}$ refer to the current length of the $1^{\text {st }}$ adherend and the length decomposed into $x$ and $z$ components Figure $3 b$.

\section{Local Coordinate System}

First, the internal force vector and stiffness matrix will be found in the local, rotating coordinate system. The stress and strain of the adherends and adhesive are assembled together in one stress and one strain vector as shown:

$$
\hat{\boldsymbol{\sigma}}=\left[\begin{array}{lllllllll}
\hat{\boldsymbol{\sigma}}_{1} & \hat{\boldsymbol{\sigma}}_{a 1} & \ldots & \hat{\boldsymbol{\sigma}}_{i} & \hat{\boldsymbol{\sigma}}_{a i} & \ldots & \hat{\boldsymbol{\sigma}}_{N-1} & \hat{\boldsymbol{\sigma}}_{a(N-1)} & \hat{\boldsymbol{\sigma}}_{N}
\end{array}\right]^{T}
$$

and

$$
\hat{\boldsymbol{\varepsilon}}=\left[\begin{array}{lllllllll}
\hat{\boldsymbol{\varepsilon}}_{1} & \hat{\boldsymbol{\varepsilon}}_{a 1} & \ldots & \hat{\boldsymbol{\varepsilon}}_{i} & \hat{\boldsymbol{\varepsilon}}_{a i} & \ldots & \hat{\boldsymbol{\varepsilon}}_{N-1} & \hat{\boldsymbol{\varepsilon}}_{a(N-1)} & \hat{\boldsymbol{\varepsilon}}_{N}
\end{array}\right]^{T}
$$

where the overbar caret denotes quantities in the local rotating coordinate system. The local stress and strain vectors for the $i$ th adherend, $\hat{\boldsymbol{\sigma}}_{i}$ and $\hat{\boldsymbol{\varepsilon}}_{i}$, contain only the axial compenents of stress/strain in the $x$-direction, $\hat{\sigma}_{i}$ and $\hat{\varepsilon}_{i}$. The local stress and strain vectors for the $i$ th adhesive, $\hat{\boldsymbol{\sigma}}_{a i}$ and $\hat{\boldsymbol{\varepsilon}}_{a i}$, contain peel and shear compenents of the stress, $\hat{\sigma}_{a i}$ and $\hat{\tau}_{a i}$, and the strain, $\hat{\varepsilon}_{i}$ and $\hat{\gamma}_{a i}$. Using beam theory and assuming small strains from the rotated coordinate system, the strains are related to the adherend centerline displacements, $\hat{\mathbf{u}}$, by the equation

$$
\hat{\boldsymbol{\varepsilon}}=\mathbf{G} \hat{\mathbf{u}}
$$

where the adherend centerline displacements are a collection of centerline displacement vectors for each adherend layer given as

$$
\mathbf{u}=\left[\begin{array}{lllll}
\mathbf{u}_{1}^{T} & \ldots & \mathbf{u}_{i}^{T} & \ldots & \mathbf{u}_{N}^{T}
\end{array}\right]^{T}
$$

and the centerline displacement vector of adherend $i$ is given as

$$
\mathbf{u}_{i}=\left[\begin{array}{llllll}
u_{i}(x) & u_{i}(x)_{, x} & w_{i}(x) & w_{i}(x)_{, x} & w_{i}(x)_{, x x} & w_{i}(x)_{, x x x}
\end{array}\right]^{T} .
$$


where $u$ and $w$ are $x$ - and $z$-direction displacements of the adherend centerline and the subscript,$x$ denotes the derivative with respect to $x$. Additionally, $\mathbf{G}$ is an assembly of the contributions of the adherend and adhesive layers assembled in the form

$$
\mathbf{G}=\left[\begin{array}{lllll}
{\left[\mathbf{G}_{1}\right]} & & & & \\
{\left[\mathbf{G}_{a 1}\right]} & & & & \\
& \ddots & & & \\
& & {\left[\mathbf{G}_{i}\right]} & & \\
& & {\left[\mathbf{G}_{a i}\right]} & & \\
& & \ddots & \\
& & & {\left[\mathbf{G}_{N-1}\right]} \\
& & & {\left[\mathbf{G}_{a(N-1)}\right]} \\
& & & & {\left[\mathbf{G}_{N}\right]}
\end{array}\right]
$$

where the sub-matrices are defined as .

$$
\mathbf{G}_{i}=\left[\begin{array}{cccccc}
0 & 1 & 0 & 0 & -z_{i} & 0 \\
0 & 0 & 0 & 0 & 0 & 0
\end{array}\right]
$$

and

$$
\mathbf{G}_{a i}=\left[\begin{array}{cccccccccccc}
0 & 0 & \frac{1}{\eta_{a i}} & 0 & 0 & 0 & 0 & 0 & \frac{-1}{\eta_{a i}} & 0 & 0 & 0 \\
\frac{1}{\eta_{a i}} & 0 & 0 & \frac{t_{i}}{2 \eta_{a i}} & 0 & 0 & \frac{-1}{\eta_{a i}} & 0 & 0 & \frac{-t_{i+1}}{2 \eta_{a i}} & 0 & 0 \\
0 & 0 & 0 & 0 & 0 & 0 & 0 & 0 & 0 & 0 & 0 & 0
\end{array}\right]
$$

Furthermore, since the deflections in the local, rotated coordinate system are considered small, the shape functions, $\mathbf{N}$, derived for the linearly elastic case are used ${ }^{7-9}$. Using the shape functions for the linear case, the local strain and displacements in the rotated coordinate system are related by the equation

$$
\hat{\boldsymbol{\varepsilon}}=\mathbf{B} \hat{\mathbf{q}}
$$

where $\mathbf{B}$ is defined as

$$
\mathbf{B}=\mathbf{G N} .
$$

The principle of virtual work of the element can be written as:

$$
\delta\left(W^{I n t}-W^{E x t}\right)=0
$$

and the internal work can be written as the internal nodal forces multiplied by the nodal virtual displacements, or the integral of the strain energy density over the volume of the element:

$$
\delta W^{I n t}=\delta \hat{\mathbf{q}}^{T} \hat{\mathbf{f}}^{I n t}=\int_{V} W(\delta \hat{\mathbf{q}}) d V
$$


where $V$ is the volume of the element, and $W(\delta \hat{\mathbf{q}})$ is the strain energy density of the element resulting from a virtual displacement. Since the deformations are small relative to the rotated coordinate system, the internal virtual work can be rewritten as

$$
\delta W^{I n t}=\int_{V} \delta \hat{\mathbf{q}}^{T} \mathbf{B}^{T} \hat{\boldsymbol{\sigma}} d V
$$

Assuming that external forces only occur as nodal forces and moments, the external virtual work of the element becomes,

$$
\delta W^{E x t}=\delta \hat{\mathbf{q}}^{T} \hat{\mathbf{f}}^{E x t}
$$

Finally, using Equation 26, and noting the fact that the virtual displacements are arbitrary, the resulting equilibrium equation is,

$$
\int_{V} \mathbf{B}^{T} \hat{\boldsymbol{\sigma}} d V=\hat{\mathbf{f}}^{E x t}
$$

Now, the local internal nodal forces are

$$
\hat{\mathbf{f}}^{I n t}=\int_{V} \mathbf{B}^{T} \hat{\boldsymbol{\sigma}} d V
$$

with the local stiffness matrix being given by

$$
\hat{\mathbf{k}}=\int_{V} \mathbf{B}^{T} \mathbf{D B} d V
$$

where the stiffness matrix, $\mathbf{D}$, is given as,

$$
\mathbf{D}=\frac{d \hat{\boldsymbol{\sigma}}}{d \hat{\boldsymbol{\varepsilon}}}
$$

Note that for linear elastic materials, the integration can be carried out analytically, resulting in a reduced equation which only requires integration in $x$.

\section{Global Coordinate System}

Now we seek to find the residual and the stiffness matrix in the global coordinate system. Since the internal work is not dependant on the frame of reference, one can write

$$
\delta \mathbf{q}^{T} \mathbf{f}^{I n t}=\delta \hat{\mathbf{q}}^{T} \hat{\mathbf{f}}^{I n t}
$$

where the nodal virtual displacements in the global frame are related to those in the local rotated coordinate frame through the equation:

$$
\delta \mathbf{q}=\delta \mathbf{q}_{\text {rig }}+\mathbf{T} \delta \hat{\mathbf{q}}
$$

making Equation 34

$$
\delta \mathbf{q}^{T} \mathbf{f}^{I n t}=\left(\delta \mathbf{q}^{T}-\delta \mathbf{q}_{r i g}{ }^{T}\right) \mathbf{T}^{T} \hat{\mathbf{f}}^{\text {Int }}
$$

Since rigid body motion does not result in the generation of internal forces, 


$$
\delta \mathbf{q}_{r i g}{ }^{T} \mathbf{T}^{T} \hat{\mathbf{f}}^{I n t}=0
$$

and Equation 36 becomes

$$
\delta \mathbf{q}^{T} \mathbf{f}^{I n t}=\delta \mathbf{q}^{T} \mathbf{T}^{T} \hat{\mathbf{f}}^{I n t}
$$

With the virtual displacements being arbitrary, the internal nodal force vector in the global coordinate system becomes

$$
\mathbf{f}^{I n t}=\mathbf{T}^{T} \hat{\mathbf{f}}^{I n t} .
$$

To find the global tangent stiffness matrix, differentiation of Equation 36 gives

$$
\delta \mathbf{f}^{I n t}=\delta \mathbf{T}^{T} \hat{\mathbf{f}}^{I n t}+\mathbf{T}^{T} \delta \hat{\mathbf{f}}^{\text {Int }} .
$$

The second term in the above equation becomes

$$
\mathbf{T}^{T} \delta \hat{\mathbf{f}}^{I n t}=\mathbf{T}^{T} \mathbf{k} \delta \hat{\mathbf{q}}=\mathbf{T}^{T} \hat{\mathbf{k}} \mathbf{T} \delta \mathbf{q}-\mathbf{T}^{T} \hat{\mathbf{k}} \mathbf{T} \delta \mathbf{q}_{r i g}
$$

The last term vanishes because, as before, displacements resulting in rigid body translation and rotation do not generate any internal force. The first term on the right side of Equation 40 is more difficult to obtain. The difficulty lies in the fact that $\mathbf{T}$ contains sines and cosines of $\phi$, which in turn contain $\mathbf{q}^{1}$ and $l$. However, Crisfield ${ }^{15}$ provides an approximation, which assumes that the extension $\delta l$ is small. Based on this assumption, the first term in Equation 40 can be rewritten as

$$
\delta \mathbf{T}^{T} \hat{\mathbf{f}}^{I n t}=\mathbf{T}_{, \phi}^{T} \hat{\mathbf{f}}^{I n t} \phi_{, \mathbf{q}} \delta \mathbf{q}
$$

where

$$
\mathbf{T}_{, \phi}=\left[\begin{array}{lll}
\mathbf{T}_{1, \phi} & & \\
& \ddots & \\
& & \mathbf{T}_{1, \phi}
\end{array}\right]
$$

and

$$
\mathbf{T}_{1, \phi}=\left[\begin{array}{ccc}
-s & c & 0 \\
-c & -s & 0 \\
0 & 0 & 0
\end{array}\right]
$$

Similarly,

$$
\phi_{, \mathbf{q}}=\left[\begin{array}{llll}
\phi_{1, \mathbf{q}} & 0 & \ldots & 0
\end{array}\right]
$$

and 


$$
\phi_{1, \mathbf{q}}=\frac{1}{l_{1}}\left[\begin{array}{llllll}
s & -c & 0 & -s & c & 0
\end{array}\right] .
$$

Combining all of these equations, the global tangent stiffness matrix can be written as a combination of the material stiffness, $\mathbf{k}_{\text {mat }}$, and the geometric stiffness, $\mathbf{k}_{\text {geo }}$, in the relation

$$
\mathbf{k}=\mathbf{k}_{\text {mat }}+\mathbf{k}_{\text {geo }}
$$

where

$$
\mathbf{k}_{g e o}=\mathbf{T}_{, \phi}^{T} \hat{\mathbf{f}}^{I n t} \phi_{, \mathbf{q}}
$$

and

$$
\mathbf{k}_{\text {mat }}=\mathbf{T}^{T} \hat{\mathbf{k}} \mathbf{T} .
$$

Both the geometric and material stiffness matrices are functions of the nodal displacements, making the system of equilibrium equations nonlinear. The Newton-Raphson method can be utilized to find the solution. It was already noted that one of the benefits of this method is that the formulation of the linear element, introduced before ${ }^{9}$ can be utilized. Another major advantage of this method lies in the fact that the local rotational frame stiffness and internal force vectors are not functions of the nodal displacements. Since numerical integration is used in finding these vectors/matrices, the integration must only be carried out once during the analysis. This saves a considerable amount of computational time, especially for an element like the joint element, which requires more refined integration for the higher order shape functions.

\section{B. Material Nonlinearities}

Since modern polymeric adhesives often display highly nonlinear material behavior, it was necessary to include material nonlinearities in the joint element to estimate joint strengths more correctly. A particularly simple nonlinear elastic stress law was chosen:

$$
\hat{\boldsymbol{\sigma}}=\hat{\boldsymbol{\sigma}}(\hat{\boldsymbol{\varepsilon}})
$$

where the stress is some general function of the strain. The only major change from the previous co-rotational formulation is that Equation 31 becomes

$$
\hat{\mathbf{k}}=\int_{V} \mathbf{B}^{T} \mathbf{D}(\hat{\mathbf{q}}) \mathbf{B} d V
$$

where the local stiffness matrix in the rotated coordinate system is now a function of the local displacements.

Although it would be more correct to use an incremental flow type plasticity formulation that distinguishes loading and unloading stiffness, the simple nonlinear elastic relation, which assumes no permanent plastic strain, was chosen for several reasons. While this was chosen for simplicity sake, this decision can also be justified. The joint element is meant to be a design tool to give general approximations, so it is not expected that such a tool will be used in situations requiring unloading capabilities. Additionally, the nature of adhesively bonded joints is such that the high stresses occur in concentrated form at the joint edges. Since the failing adhesive domain is eliminated in the iteration process (to be described later) the assumption of a nonlinear elastic type stress-strain law suffices for this modeling process since potential regions of "unloading" are minimal and contained in the regions which are eliminated. Thus, this assumption does lead to a meaningful rendition of the joint physics, yet facilitating an efficient (in the computational sense) solution strategy.

One other aspect worthy of discussion is the integration requirements for the nonlinear material formulation. When the adherends have a nonlinear stress-strain relationship, Equations 31 and 51 must be integrated over $\hat{x}$ and 
$\hat{z}$ at each Newton-Raphson iteration to allow a general stress-strain relationship. This causes a considerable increase in computational time. However, there are some cases when this is not necessary. If only the adhesive layers have a nonlinear stress-strain relation, integration over $\hat{z}$ can be avoided because the stress is constant through the thickness of the adhesive layer. Additionally, if the functions for the nonlinear stress-strain relations are known (and simple enough), integration over $\hat{z}$ can be accomplished analytically. However, this would mean that the formulation is only good for that specific stress-strain relation, and cannot be extended to other general relations.

\section{Crack Growth}

When some user defined failure criterion is reached in some part of the adhesive layer, that portion of the adhesive is considered "failed" and can carry no load and has no stiffness. Setting the stress and stiffness of that portion of the adhesive to zero is an easy way to model the failure of the adhesive, but the shape functions for the joint element were not originally calculated based on a joint with failed adhesive, and cannot accurately model this new situation. Therefore, as with more traditional shape function prescribed finite elements, more elements are required to accurately find the solution. In the case of failed adhesive, a great number of elements may be needed, as will be illustrated later.

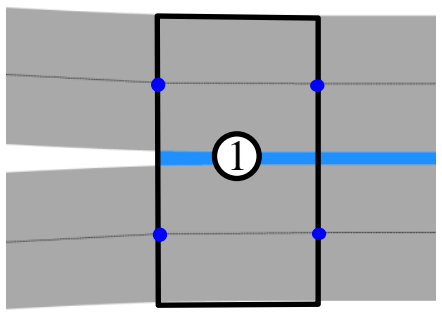

(a) Uncracked

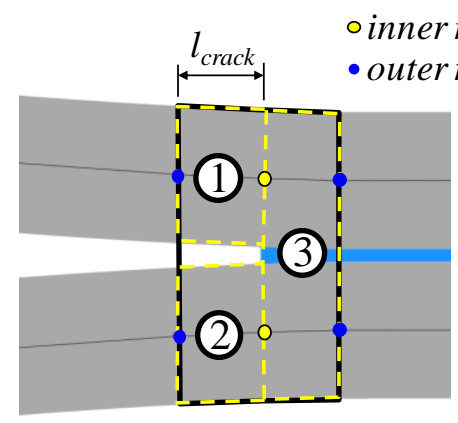

(b) Partially Cracked

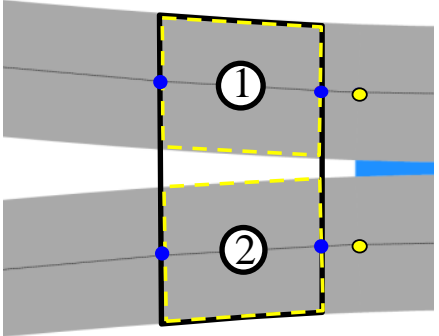

(c) Fully Cracked

Figure 4. Diagram showing a) an uncracked joint element, b) a partially cracked element, and c) a fully cracked joint element.

In order to increase the accuracy of the joint element after adhesive failure and crack growth, a method of removing the adhesive and adapting the mesh to the crack was devised. Since the joint element is meant to be used as a user defined element in a larger global assembly in commercially available finite element software, the mesh change would have to be strictly internal to the element so that the surrounding model does not have to change. Therefore, a sub-assembly method was devised to handle adhesive failure (Figure 4) and is outlined in Figure 5.

First, when failure in the adhesive is detected, the element is replaced by a sub-assembly with three elements as shown in Figure $4 \mathrm{~b}$. The length of the crack determines the lengths of the sub-assembly elements. Within a Newton-Raphson type solver, the nodal displacements are prescribed (guessed) and the stiffness and internal force vector for the element are calculated. These vectors/matrices for all of the elements in the assembly are assembled, boundary conditions and loads are applied, and the residual (error of the initial nodal displacement guess) is calculated. If the residual isn't within some tolerable state, a new nodal displacement "guess" is calculated based on the previous displacement, residual, and stiffness values and the whole cycle repeats.

In the case of a joint element with a crack, only the outer nodal displacements are prescribed since the global finite element assembly isn't aware of the existence of the sub-assembly and the inner nodes. Therefore, the subassembly becomes a nonlinear model within another nonlinear model and must be solved with its own NewtonRaphson type solution procedure. The prescribed nodal displacements of the outer nodes become the boundary conditions for the sub-assembly, and the whole system is solved using a nonlinear solver. When the desired error tolerance is reached, a stiffness matrix and internal force vector for the sub-assembly has been calculated. However, these quantities still have the inner degrees of freedom contained within. The force vector and stiffness matrix are then reduced using the Guyan Reduction Method ${ }^{16-18}$. Once the internal degrees of freedom are removed, the stiffness matrix and force vector can be considered to be that of the equivalent joint element, and can be passed on to the global assembly. 


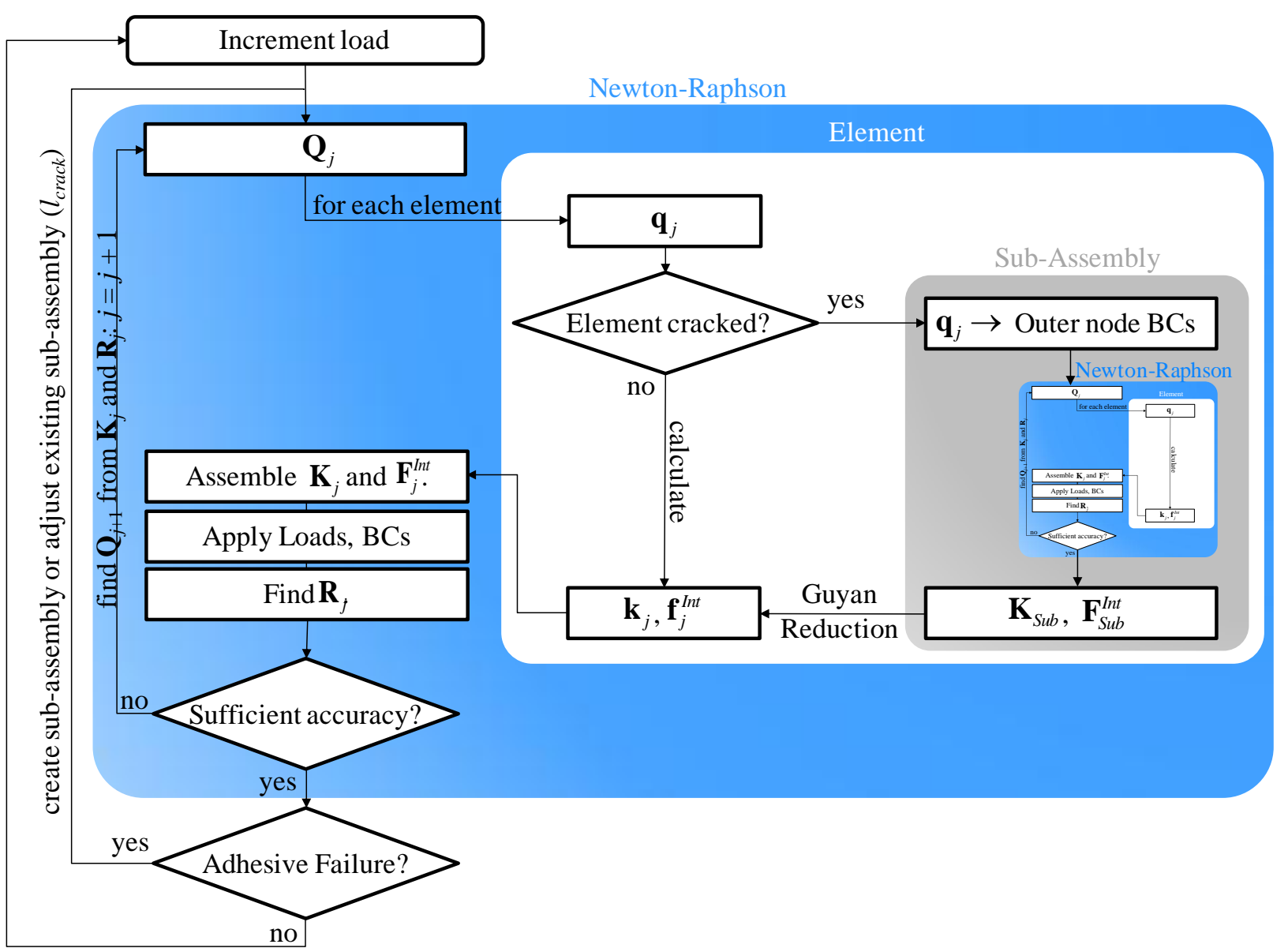

Figure 5. Flow chart showing how cracked element Sub-Assembly is incorporated into joint element solution procedure.

After the global system is solved, there is a check to see if the crack has grown, or if new adhesive failure has been detected. If this is the case, the sub-assembly is adjusted by changing the lengths of the sub-assembly elements, and the global system is re-solved. This is done until no new adhesive failure occurs and the crack is in equilibrium. A crack scaling constant, $C_{1}$, has been introduced to speed up or slow down crack growth as needed, and is used in the equation

$$
l_{\text {crack }}^{\text {cur }}=l_{\text {crack }}^{\text {cur }}-C_{1}\left(l_{\text {crack }}^{\text {cur }}-l_{\text {crack }}^{\text {prev }}\right)
$$

where $l_{\text {crack }}^{\text {prev }}$ is the previous crack length (prior to the global Newton-Raphson procedure) and $l_{\text {crack }}^{\text {cur }}$ is the current crack length. Setting $C_{1}<0$ causes the crack to grow further than detected, and is useful when multiple iterations are needed to find crack equilibrium. Setting $C_{1}>0$ causes the crack to grow less than detected, and is necessary when crack overshoot is a concern.

The advantage of this method is that fewer elements are needed in order to accurately capture crack growth. One can use the minimum elements needed to accurately capture the material and geometric nonlinear effects without crack growth being a factor. This can mean dramatically reducing the number of elements required, especially when there is little material nonlinearity, and when strains in the joint are small.

One of the major disadvantages of this method is the increased computational time. A local nonlinear problem must be solved within each iteration of the global nonlinear problem. Although the local nonlinear problem is always limited to three elements, it can significantly increase the runtime. Furthermore, the global load increment is repeated if the crack grows and the sub-assemblies need to be created or re-meshed. Although the crack scaling 
parameter can significantly help in limiting the iterations needed to find crack equilibrium this process can still be costly. However, the costs can be justified if joint strength prediction is of concern. Joint strength has been identified as a controlling factor in the ultimate load bearing capacity of many bonded structures.

\section{Adhesive Model Characterization}

One of the most important inputs for determining the strength of a joint is the characterization of the adhesive constitutive response. There have been many methods of characterizing the adhesive material, but two have emerged as the most common: bulk adhesive tensile test and fracture mechanics characterization tests (DCB, ENF, etc.). Therefore, the following sections outline methods of using both bulk adhesive tensile test data and fracture mechanics inputs to characterize the joint. Ultimately, the test data available and personal preferences of the user will decide which route to take.

\section{Bulk Adhesive Tensile Characterization}

One common way of characterizing adhesive materials is by performing tensile tests on bulk adhesive specimens, such as those depicted in Figure 6. The following section will outline an approximate method for modeling the adhesive based on such adhesive characteristic data, and will discuss the formulation and underlying assumptions involved.

(a)
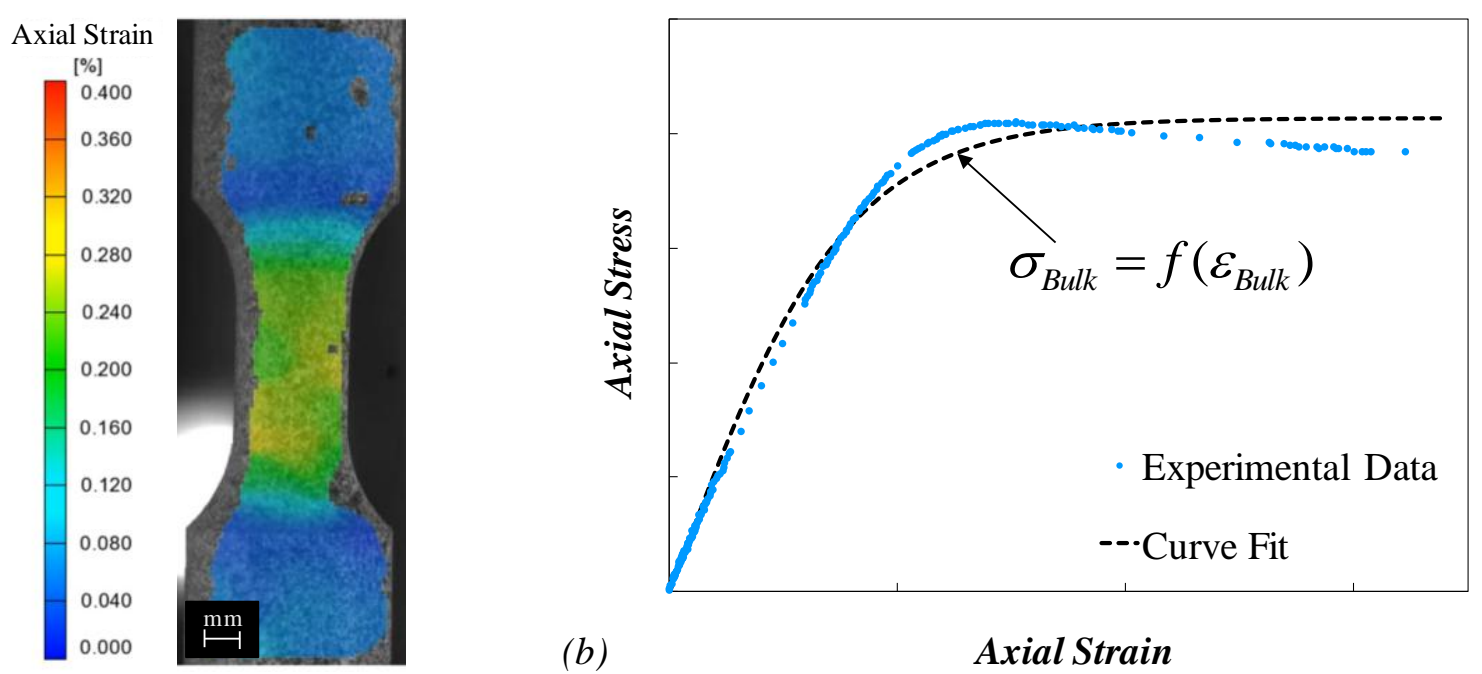

(b)

\section{Axial Strain}

Figure 6. Adhesive may be characterized by (a) experimental bulk adhesive tensile tests, then (b) fitting a curve to the stress-strain plot.

If the adhesive is much deeper than it is thick $\left(b_{a i} \ll<\eta_{a i}\right)$, it can be considered to be in a state of plane strain in the $z-x$ plane (Figure 7), and the stress-strain relation for plane strain can be applied. Furthermore, if we assume that the adhesive is perfectly bonded to the adherends and that the adherends are much stiffer than the adhesive $\left(E_{a i}<<\right.$ $\left.E_{i}\right)$, then it can be argued that the extensional strain in the adhesive is much smaller than the peel and shear components $\left(\hat{\varepsilon}_{x a i}<<\hat{\varepsilon}_{a i}, \hat{\gamma}_{a i}\right.$ ) which is the root of the assumption:

$$
\hat{\varepsilon}_{x a i} \approx 0
$$




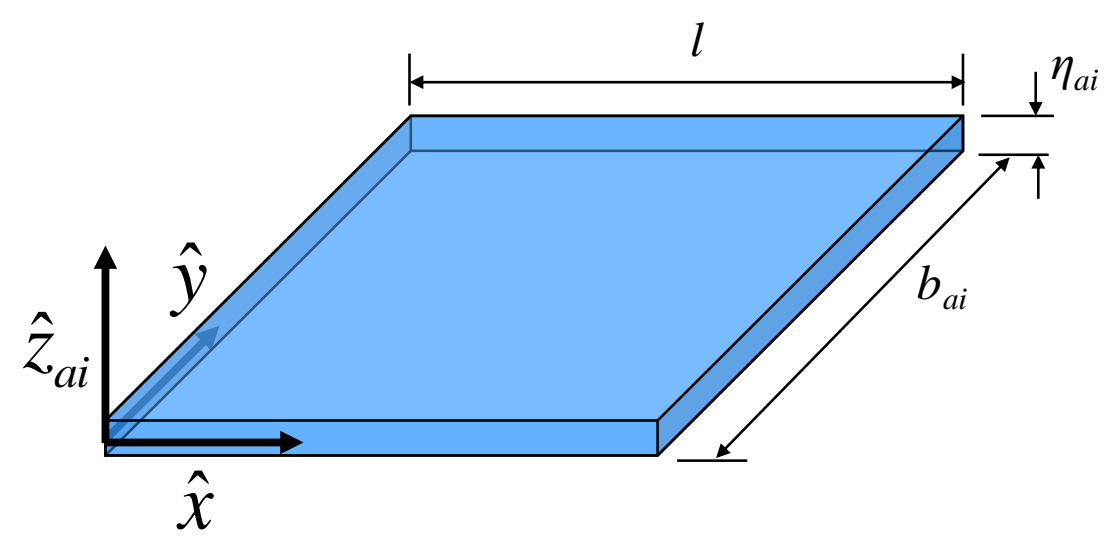

Figure 7. Assuming that the adhesive is perfectly bonded to the adherends, the adhesive can be considered a constrained body under triaxial stress.

This assumption gives rise to the common practice in adhesive joint analysis of ignoring the extensional stress and strain in the formulation. With the extensional strains being relatively small, the strain energy of the adhesive layer is virtually unaffected and does not necessarily need to be included. Although the extensional strain is negligible, the extensional stress (in both the $x$ - and $y$-directions) is not insignificant, placing the adhesive in a state of triaxial stress ${ }^{19}$. Using these assumtpions and linear elasticity, the extensional stress in the adhesive, $\hat{\sigma}_{x a i}$, can be written in terms of the peel strain:

$$
\hat{\sigma}_{x a i}=C_{2} v_{a i} \hat{\varepsilon}_{a i}
$$

where

$$
C_{2}=\frac{E_{a i}}{\left(1+v_{a i}\right)\left(1-2 v_{a i}\right)}
$$

and where $E_{a i}$ and $v_{a i}$ are the Young's modulus and Poisson's ratio of the $i$ th adhesive layer,. The axial adhesive stress can be written in terms of the peel stress:

$$
\hat{\sigma}_{x a i}=\frac{v_{a i}}{1-v_{a i}} \hat{\sigma}_{a i}
$$

The same relation is true for the extensional stress in the $y$-direction, $\hat{\sigma}_{y a i}$. This can be used to find the extensional stress without necessarily including it into the formulation. Furthermore, the peel stress becomes a function of the peel strain only:

$$
\hat{\sigma}_{a i}=\frac{1-v_{a i}}{\left(1-2 v_{a i}\right)\left(1+v_{a i}\right)} E_{a i} \hat{\varepsilon}_{a i}
$$

This shows that the effective "resistance" to deformation in the z-direction is amplified by a factor that depends on Poisson's ratio. Although this relation is intended for linear elasticity, the relation was assumed to hold for the nonlinear stress-strain relation as well. Therefore, the stress-strain relation was redefined as:

$$
\hat{\sigma}_{a i}=\frac{1-v_{a i}}{\left(1-2 v_{a i}\right)\left(1+v_{a i}\right)} f\left(\hat{\varepsilon}_{a i}\right)
$$

which effectively increases the adhesive modulus.

A Von Mises failure criterion was chosen for this particular formulation, although the same formulation could easily be altered for a different criterion ${ }^{20}$. Applying the notation for the adhesive layer, assuming the shear stresses 
in the $x y$ and $y z$ planes to be negligible, and using Equation 56, the Von Mises equivalent stress in terms of the shear and peel stress components are

$$
\sigma_{v m}^{2}=\left(\frac{1-2 v_{a i}}{1-v_{a i}}\right)^{2} \sigma_{a i}{ }^{2}+3 \tau_{a i}{ }^{2}
$$

Although the Von Mises equivalent stress is normally used to find the yield stress, in this case it will be assumed to hold for the entire nonlinear adhesive stress/strain response. Therefore, the Von Mises equivalent stress for a certain adhesive will be a nonlinear function of the adhesive strain found using bulk adhesive tensile tests (Figure 6a):

$$
\sigma_{v m}=\sigma_{\text {Bulk }}=f\left(\varepsilon_{\text {Bulk }}\right) \text {. }
$$

To find the nonlinear curves approximating the peel and shear stress in the adherend, one more relation must be defined. A new variable will be introduced, $\psi_{i}$, which represents the ratio of peel to shear stress for adhesive layer $i$ of a particular joint configuration:

$$
\psi_{i}=\frac{\hat{\sigma}_{a i}}{\left|\hat{\tau}_{a i}\right|}
$$

allowing the shear stress to be defined as a function of the bulk stress:

$$
\hat{\tau}_{a i}^{2}=\frac{\sigma_{B u l k}^{2}}{3+\left(\frac{1-2 v_{a i}}{1-v_{a i}}\right)^{2} \psi_{i}^{2}} .
$$

The method of finding the strain was a bit more arbitrary. Others have done this by utilizing a Von Mises strain criterion or similar methods ${ }^{5,11,21}$. For the current formulation, it was assumed that the bulk adhesive tensile specimen strain and the adhesive layer strains were linearly related to each other through the equations

$$
\hat{\gamma}_{a i}=C_{3} \varepsilon_{B u l k}
$$

and

$$
\hat{\varepsilon}_{a i}=C_{4} \varepsilon_{B u l k}
$$

where the constants $\mathrm{C}_{3}$ and $\mathrm{C}_{4}$ are found such that the initial slopes of the shear and peel stress-strain curves become the normal and shear modulus respectively.

For an actual joint, the ratio of peel to shear stress, $\psi_{i}$, not only varies across the joint, but changes during loading due to nonlinear geometric effects and nonlinear material effects. Therefore, this value will in actuality be a function of the joint geometry, loading, materials, and location within the adhesive in question. However, to simplify the determination of this value, it is proposed that one assume that the ratio of peel to shear doesn't change significantly during the loading event and that only the stress at ends of the joint where the stress concentrations reside is important. The correctness of this first assumption will be tested later. Therefore, this value can be approximated by taking the ratio of the maximum peel to shear stress of the linearly elastic case as illustrated in Figure 8 . 


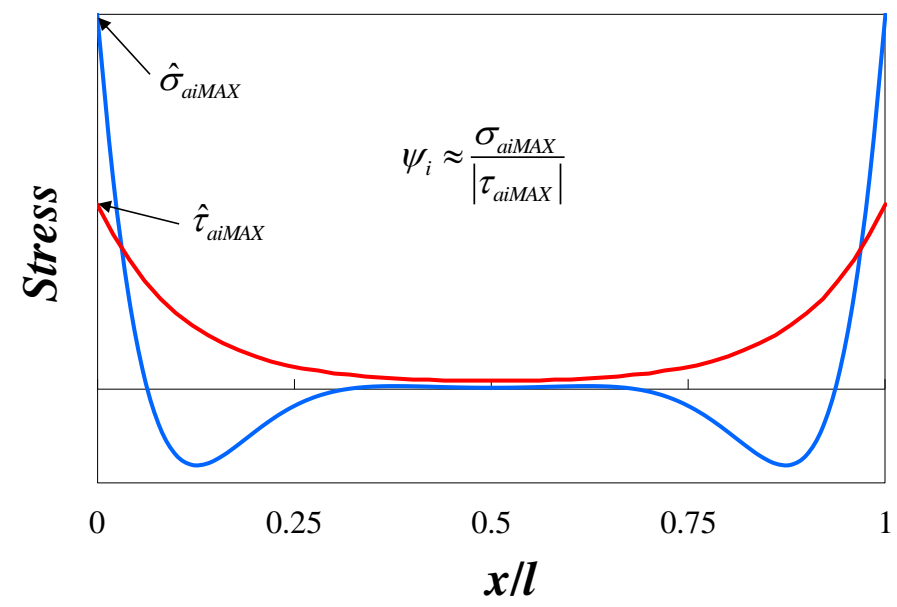

Figure 8. The peel to shear ratio for adhesive $i$ can be approximated by dividing the maximum peel stress by the maximum shear stress for the linear elastic adhesive case.

For balanced joints with the same adherend materials and geometries, the maximum occurs on both ends of the adhesive and is identical on either end. However, for unbalanced joints, the stress concentrations at the ends of the adhesive can be of unequal magnitude. Finding the peel to shear ratio based on the higher and lower of the two stress concentrations can provide a good upper and lower bound to the nonlinear solution.

In order to approximate the Von Mises failure criterion for uncoupled shear and peel, an uncoupled strain-based criterion was chosen that simply considered the adhesive failed when

$$
\frac{\hat{\varepsilon}_{a i}}{\varepsilon_{c}}=1 \text { or } \frac{\hat{\gamma}_{a i}}{\gamma_{c}}=1
$$

where $\varepsilon_{c}$ and $\gamma_{c}$ are critical peel and shear strain values. These values are found by applying Equations 63 and 64 to the maximum strain of the bulk adhesive tensile test data.

Though it might seem unusual to use a strain-based criterion to approximate the Von Mises stress, it should be kept in mind that a Von Mises yield criterion was already applied to get from the bulk adhesive tensile test data to the peel and shear stress-strain relations. If the peel to shear ratio, $\psi_{i}$, was chosen correctly, both the shear and peel components should be close to their respective critical values at the same time.

\section{Fracture Mechanics Characterization}

The joint element model is very similar to the Cohesive Zone Models (CZM) ${ }^{10,22-24}$ and is inherently suited for fracture mechanics-type inputs. One of the main differences between most mainstream cohesive zone models and the joint element adhesive model lies in the thickness of the cohesive zone. Most CZM's have no thickness, and lie at the interface between continuum elements. Since it has no thickness, a traction-separation law rather than a stress-strain law is defined for the CZM. Thus, cracks in the center of the adhesive layer can be differentiated from cracks at the interface by placing CZM elements at different locations within the adhesive, although this is computationally very costly. The joint element, on the other hand, resembles a cohesive zone with an explicit thickness. The entire adhesive layer is a single cohesive zone, and cracks in the middle of the adhesive are not differentiated from those at the interface. The traction-separation law can be transferred approximately to a stressstrain law by dividing the separation by the thickness as shown in Figure 9.

For this type of adhesive characterization, the shear and peel responses are isolated and characterized in a series of experiments ${ }^{25}$. The peel and shear responses are considered to be uncoupled and depend solely on the vertical and horizontal separations of the adherends respectively. Typically, a critical stress and fracture toughness are identified for Mode I and Mode II. Since the joint element model does not have continuum elements to represent the adhesive, it is recommended that the initial slopes of the stress-strain laws be set to the elastic modulus for peel and shear.

Finally, adhesive failure can be defined as occurring when 


$$
\left(\frac{G_{I}}{G_{I C}}\right)^{i}+\left(\frac{G_{I I}}{G_{I I C}}\right)^{j}=1 .
$$

The values of $i$ and $j$ can be chosen based on the preference of the element user.

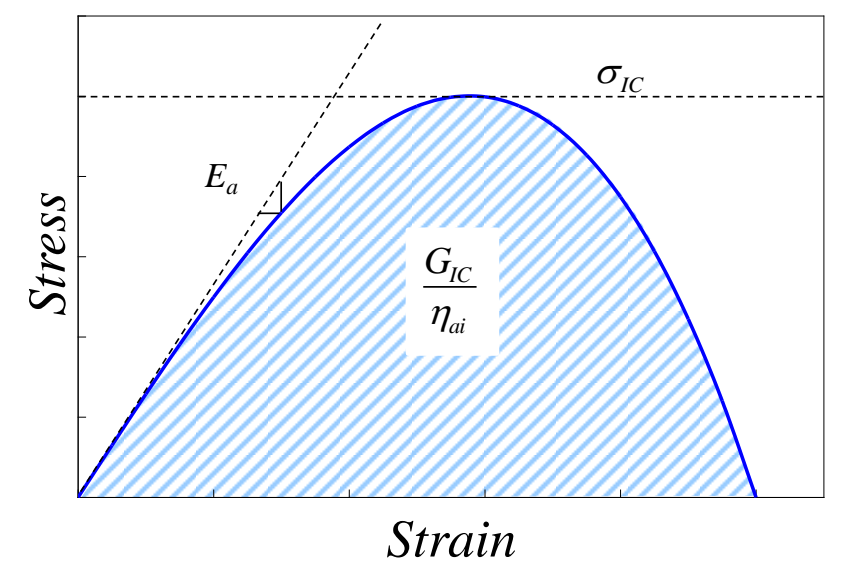

Figure 9. Fracture mechanics properties such as critical stress and fracture toughness can be used to form an adhesive stress-strain law for the joint element.

\section{Results and Validation}

\section{A. Geometric Nonlinearities}

To validate the co-rotational formulation, several example joint configurations were analyzed using the joint element and compared with 2-D dense mesh finite element solutions with nonlinear geometric effects to demonstrate the joint element's ability to capture large rotation situations and to show how many elements are typically required.

The first example was an unbalanced single overlap joint, using the joint elements with a single adhesive layer and two adherends. The unbalanced single lap joint illustrated in Figure 10 was pulled in a displacement-controlled manner. The adherends were titanium ( $E=110 \mathrm{GPa})$ and aluminum ( $E=70 \mathrm{GPa})$, with EA 9394 as the adhesive layer ( $E=4 \mathrm{GPa}, G=1.79 \mathrm{GPa}$ ). As before, the shallow width of the joint required the use of a plane stress joint element formulation and the use of 2-D plane stress elements for the dense 2-D finite element mesh model. The joint element model had 40 beam elements with one joint element, while 154,000 elements were used for the 2-D dense mesh model (Figure 11a). A comparison of the load-displacement plots of the different models is shown in Figure $11 \mathrm{~b}$. The joint element model was able to replicate the response quite well, even with only one beam element rather than 40. The actual joint region requires fewer elements because all of the bending takes place outside of the overlap region. The increased flexural rigidity of the overlap region causes it to rotate rigidly rather than bend. Therefore, more elements are required outside the overlap regions to capture the nonlinear geometric effects of the joint. 


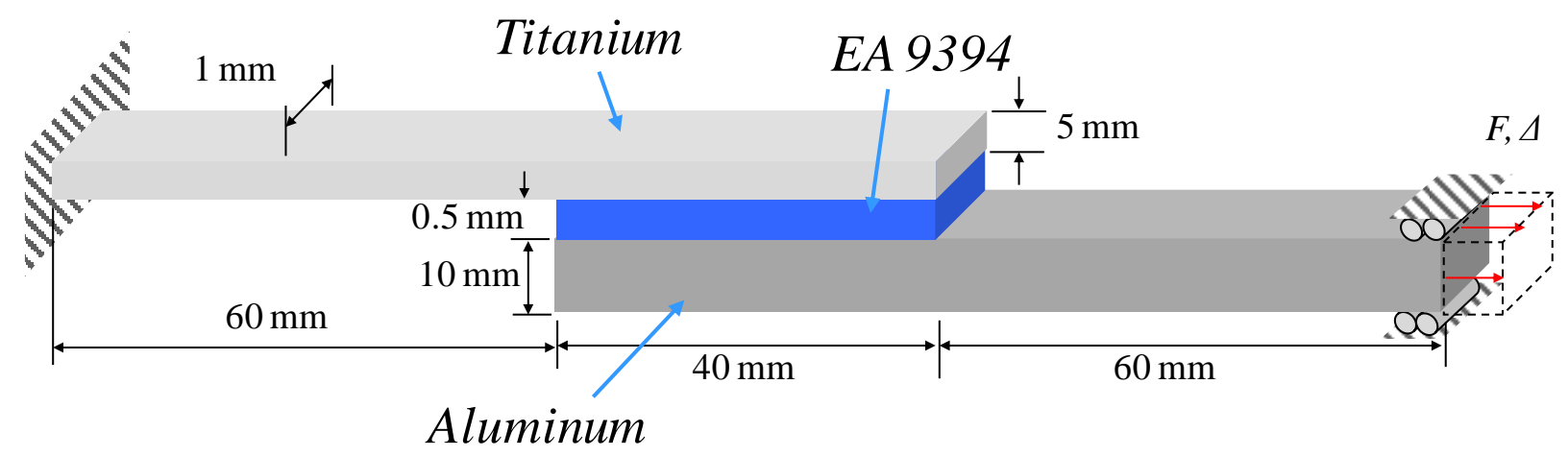

Figure 10. Single lap joint used to validate joint element co-rotational formulation.
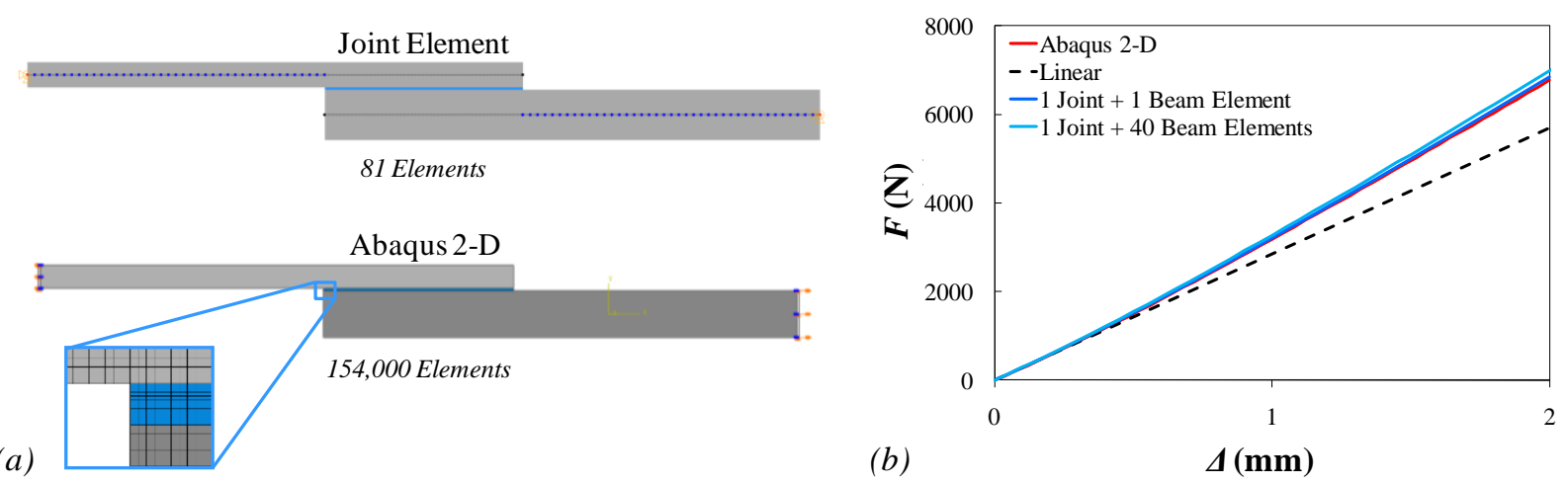

Figure 11. Comparison of (a) joint element and 2-D dense mesh finite element representation of unbalanced single lap joint and (b) the resulting load vs displacement plot.

\section{B. Material Nonlinearities}

The material nonlinearity examples featured in this section only highlight adherend nonlinearity and adhesive nonlinearity separately to show the strengths and weaknesse. Tthe limitations and abilities of the joint element in modeling nonlinear adherends are shared by beam elements in general, and more in-depth discussion on these limitations and how to overcome them are dealt with extensively in literature ${ }^{26-33}$.

The example of adherend material nonlinearity is the single lap joint shown in Figure 10, but with elasticperfectly plastic adherends. The yield stress for the titanium was set at $1050 \mathrm{MPa}$, and the aluminum was at 300 $\mathrm{MPa}$. Large rotations were considered in the analysis, and the adhesive was given linear material properties to isolate the effect of nonlinear adherends. Figure 12a shows the load-displacement plot for the joint element model using different numbers of elements. As can be seen, none of the models are that far off of each other, but more elements are certainly necessary for a converged solution. However, the load-displacement plot did not resemble that of the Abaqus 2-D dense mesh model shown in Figure 12b. The load predicted by the 2-D dense mesh model drops after a peak, whereas the joint element model does not drop, but continues to hold more load. There are two explanations for this. First, since the stress-strain relation for the adherend is nonlinear elastic, unloading of the adherends is inaccurate. When the adherends first yield, the strain increases dramatically at one point (localization) while the rest of the adherend unloads. Since unloading is inaccurately captured in the joint element model, it continues to increase in load. The second discrepancy is that beam models still have the assumption that the displacement and strain vary linearly in the $z$-direction. Since this is not the case after yielding, the model is inaccurate after initial yielding. 

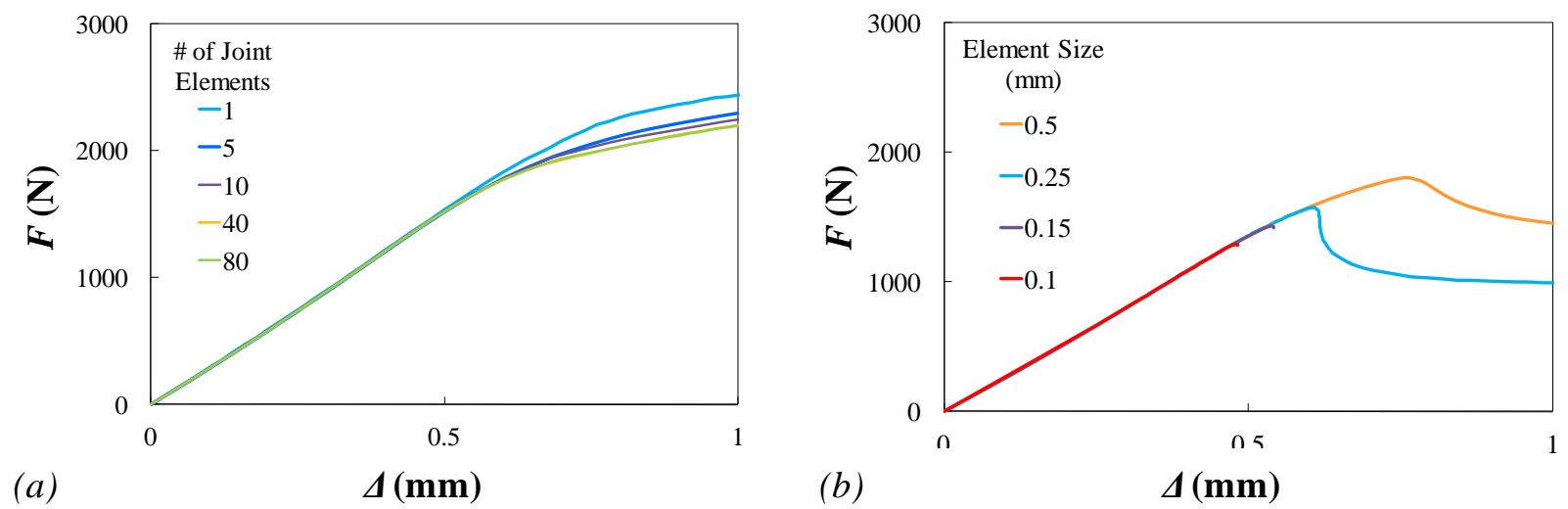

Figure 12. Plots showing the effect of element size on the load-displacement response of the single lap joint featured in Figure 10 with nonlinear adherends for the (a) joint element, and (b) dense 2-d finite element mesh.

Another observation about the 2-D dense mesh finite element model is that the solution continues to change when the element size is reduced. This is due to the stress singularity at the reentrant corners. As the element size is decreased, the stress concentration rises and the adherends yield sooner and more dramatically. Furthermore, the solution cuts off after the peak for an element size of 0.15 and $0.1 \mathrm{~mm}$. This cutoff was due to the commercial FE analysis software, which ends the analysis after the step size has become too small. This is also probably due to the stress singularity at the reentrant corners, and illustrates some of the potential difficulties of modeling joints.

This example illustrates why one should avoid using the joint element when failure of the joint is dominated by adherend yielding. It also brings out the need of applying some of the measures adopted for beam elements to the joint element to better capture the material softening of the adherends.

The second example, illustrating nonlinear adhesive, is the same joint discussed previously, except with linear adherends and a nonlinear adhesive stress-strain relation. The adhesive had an elastic-perfectly plastic bulk adhesive tensile test stress-strain relation with the linear properties being that of EA 9394 ( $E=4 \mathrm{GPa}, G=1.5 \mathrm{GPa}$ ) and the bulk yield stress was $40 \mathrm{MPa}$. The procedure outlined previously was followed to find the peel and shear yield stress, $\sigma_{a Y}$ and $\tau_{a Y}$. The adhesive was allowed to yield indefinitely so that no crack would form or grow. This is an upper-bound prediction of joint strength according to the global yielding criterion proposed by Crocombe ${ }^{34}$.

Since the joint was unbalanced, two peel to shear ratios were found; one on each side of the adhesive. The left side was the side with the greatest magnitude of adhesive stress, while the right side was a bit lower. Since the adhesive can yield indefinitely, the maximum load will not be reached until both sides of the adhesive begin to yield. Therefore, it was expected that the peel to shear ratio of the right side, the last side to yield, would result in the most realistic solution. The peel to shear ratios and peel and shear yield stresses, along with predicted joint strengths, are shown in Table 1. A comparison of the load-displacement response using the peel to shear ratio from the left (high ratio) and the right (low ratio) is shown in Figure 13a. This is expected to provide bounds for the solution.

The load-displacement plot for different sizes of elements using the 2-D dense mesh model is shown in Figure $13 \mathrm{~b}$, while the same plot for different numbers of joint elements with is shown in Figure 13b. As with the nonlinear adherend solution (Figure 12), the reentrant corners caused stress singularities, which cause the solution to be mesh dependent for the 2-D dense mesh model. However, it appears that for the element sizes shown, the joint element predictions provide an upper and lower bound for the 2-D dense mesh solution. 

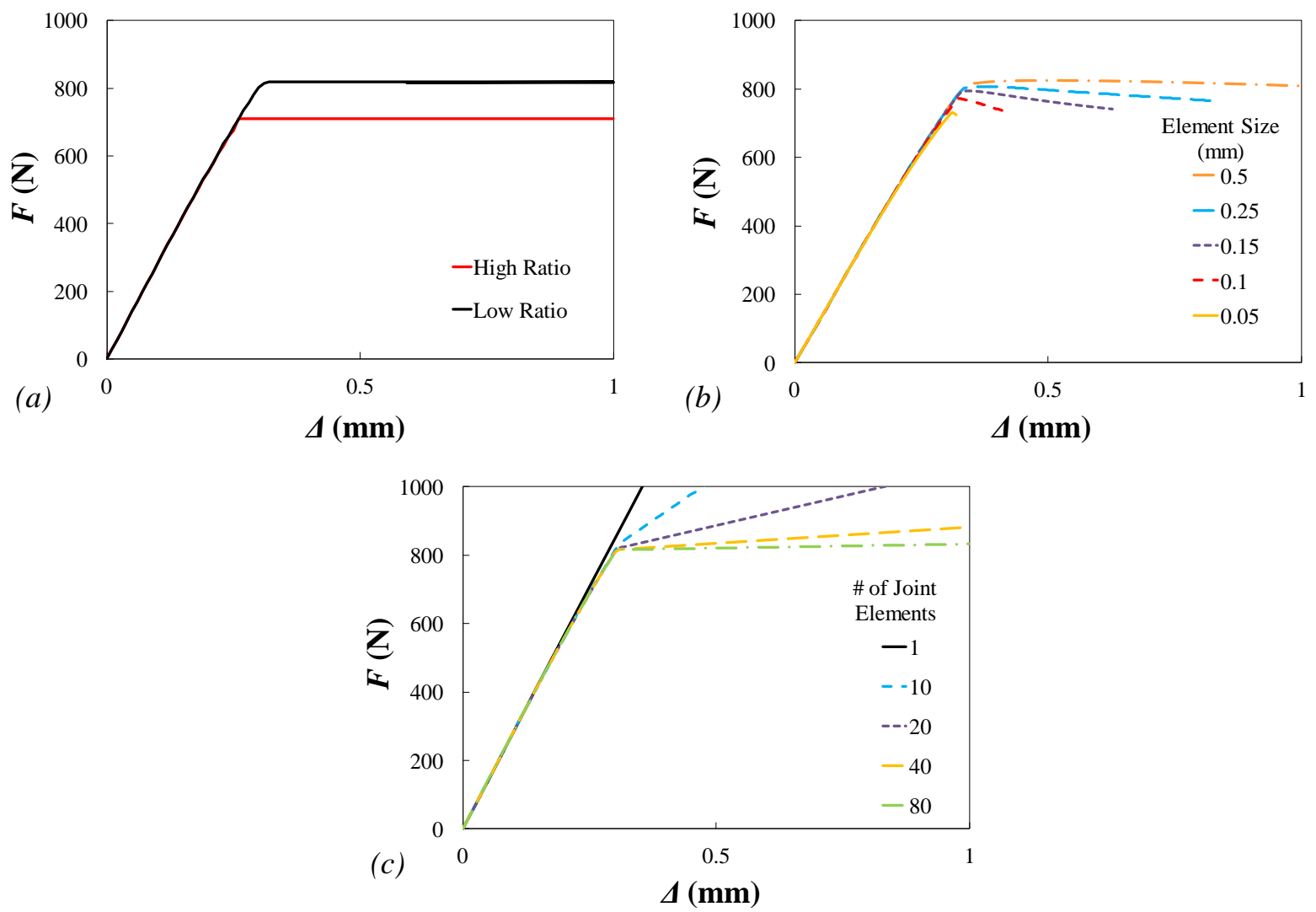

Figure 13. Load displacement plots for the joint depicted in Figure 10 with an elastic perfectly plastic adhesive with yield stress of $40 \mathrm{MPa}$. Plots show (a) the results of basing the constitutive properties on the peel to shear ratio of the highest stressed side and the lower side, and elemental convergence for the (b) 2-D dense mesh model, and (c) joint element model.

Table 1. For an unbalanced joint, the peel to stress ratio is different on each side and produces a different strength prediction.

\begin{tabular}{cccccc}
\hline $\begin{array}{c}\text { Side of } \\
\text { Adhesive }\end{array}$ & $\begin{array}{c}\text { Stress } \\
\text { Concentration }\end{array}$ & $\psi$ & $\begin{array}{c}\sigma_{a Y} \\
(\mathrm{MPa})\end{array}$ & $\begin{array}{c}\tau_{a Y} \\
(\mathrm{MPa})\end{array}$ & $\begin{array}{c}\text { Predicted Strength } \\
(\mathrm{kN})\end{array}$ \\
\hline Left & Higher & 1.63 & 29.0 & 17.1 & 710 \\
Right & Lower & 1.04 & 21.3 & 20.5 & 819 \\
\hline
\end{tabular}

\section{Crack Growth}

To illustrate the benefits of growing a crack by re-meshing rather than just setting the failed adhesive stiffness and stress to zero, a bi-layered beam was pulled apart as shown in Figure 14a. The beam was $5 \mathrm{~mm}$ wide, and the adherends had a stiffness of $100 \mathrm{GPa}$. The adhesive had a Young's modulus of $1 \mathrm{GPa}$, and was linear up to failure, which occurred at $500 \mathrm{MPa}$ (see Figure 14b). The simplistic linear-until-failure adhesive was chosen because an analytical solution can be found and because it allows crack growth without material nonlinearity, isolating this aspect of the joint element. 


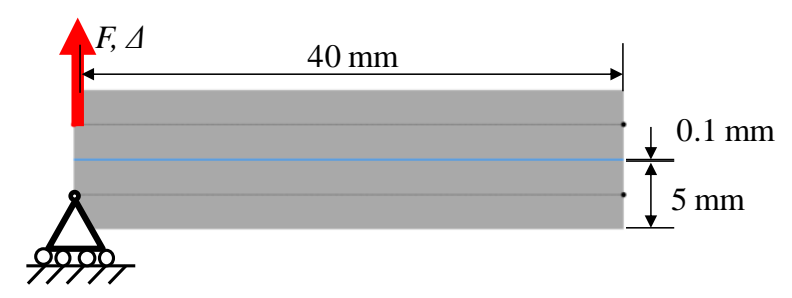

(a)

(b)

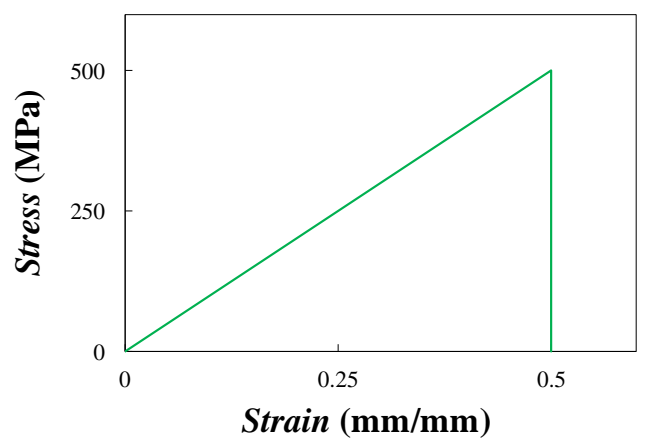

Strain $(\mathbf{m m} / \mathbf{m m})$

Figure 14. Example of the peeling of a (a) layered beam where the adhesive is modeled as (b) linear until failure to demonstrate the joint element crack growth ability.

Two different models were compared to show the benefits of re-meshing. First, rather than removing the adhesive and re-meshing, the stress and stiffness of the adhesive were simply set to zero when the stress reached 500 MPa. Second, the failed adhesive was removed and the element was replaced by a sub-assembly as illustrated in Figure 4. The results of the two models with different ways of handling crack growth are shown in Figure 15. The benefits of re-meshing are clear. For the first model, the post-peak solution oscillates around the analytical solution with the oscillation amplitude reducing for more elements. The second model with the re-meshing, on the other hand, is extremely close to the analytical solution with just a single element. There is some oscillation after the peak, but this is suspected to be caused by crack overshoot. This effect, however, disappears entirely with only four elements. This example dramatically shows that re-meshing the element to represent crack growth can result in huge elemental savings over zeroing the adhesive stiffness.
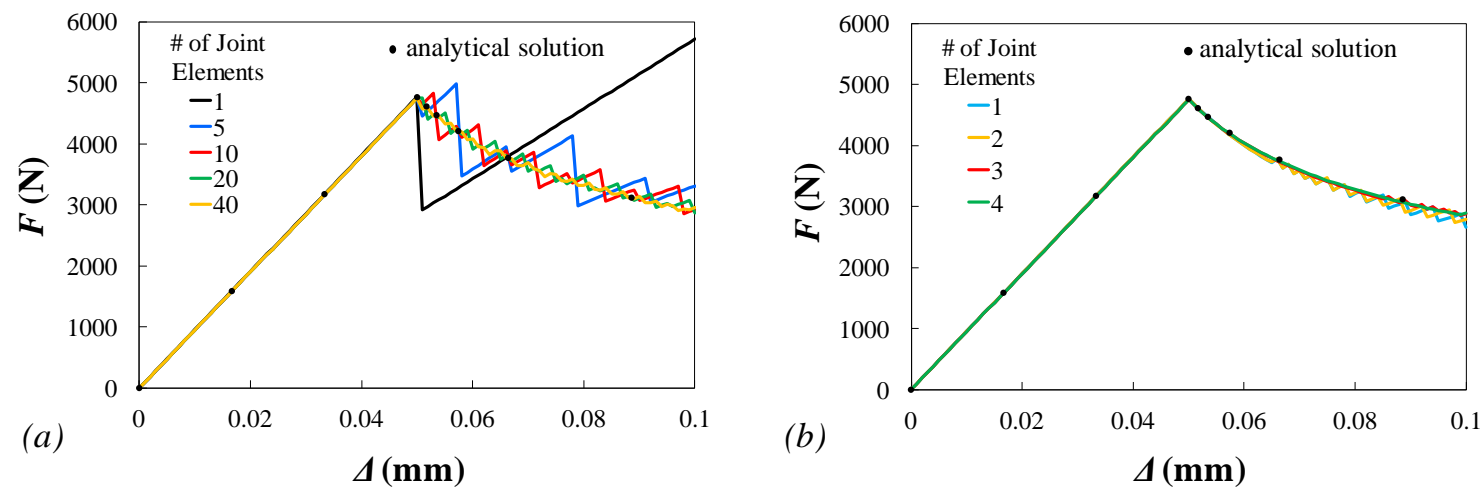

Figure 15. Load displacement plots for the peeling of a layered beam with different numbers of joint elements using (a) no re-meshing and (b) re-meshing.

\section{Experimental Validation}

Lastly, the joint element was compared with experimental data published by Harris and Adams ${ }^{11}$ on single lap joints. The tests were carried out according to ASTM D1002-72 specifications. The geometric parameters are shown in Figure 16. The adhesive was MY750 and three different aluminum alloys served as the adherends. The only difference between the alloys was the $0.2 \%$ proof stress, as shown in

Table 2. The adherends were modeled with an elastic-perfectly plastic stress-strain relation. The adhesive, MY750, was characterized using bulk adhesive tensile tests, and the bulk adhesive stress-strain relation is shown in Figure 17a. 


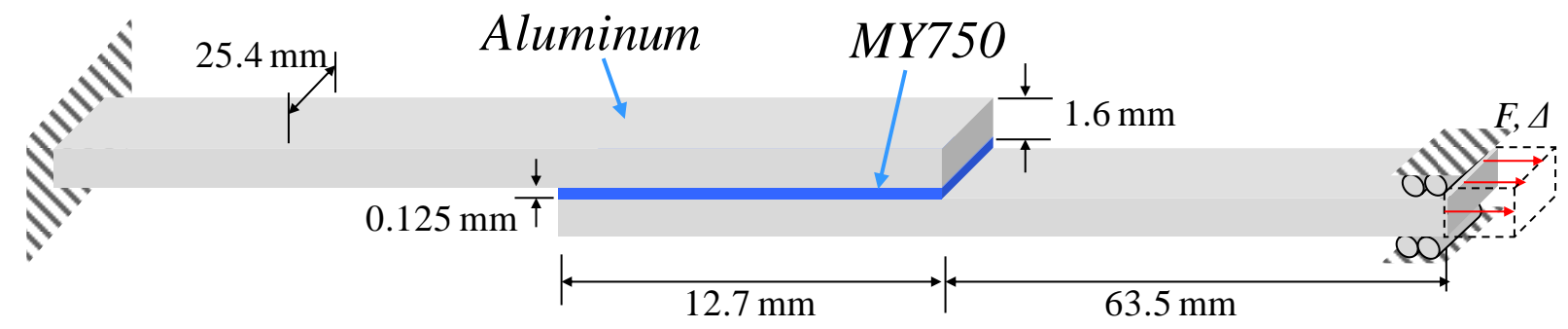

Figure 16. Geometric parameters for single lap joint tested by Harris and Adams ${ }^{11}$.

Table 2. Material properties of the single lap joint adherends and adhesive ${ }^{11}$.

\begin{tabular}{cccc}
\hline & E (GPa) & $v$ & $0.2 \%$ Proof Stress (MPa) \\
\hline MY750 & 3.44 & 0.4 & - \\
Aluminum 2L73 & 70 & 0.34 & 430 \\
Aluminum BB2hh & 70 & 0.34 & 220 \\
Aluminum BB2s & 70 & 0.34 & 110 \\
\hline
\end{tabular}

The method outlined in previously was followed to find the adhesive peel and shear stress-strain relation. First, the joint was analyzed with linear material properties and small rotations, and the peel to shear ratio, $\psi$ was found to be 1.4. Using this value, the Young's modulus, and the Poisson's ratio, the bulk adhesive tensile data was converted to the peel and shear stress-strain relations shown in Figure 17a. Using this, the joint was modeled with 20 beam elements and one joint element and was loaded in a displacement controlled manner until the peak load had been reached. The load-displacement plots for the single lap joints with different aluminum alloys are shown in Figure 17b, and the results are compared with the experimental values found by Harris and Adams ${ }^{11}$ in Table 3.

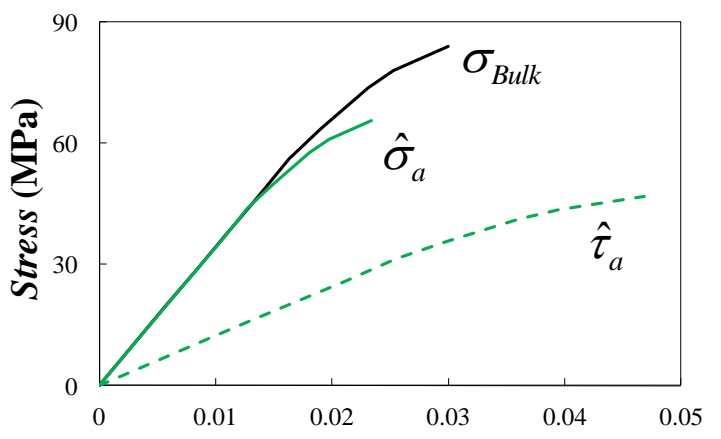

(a)

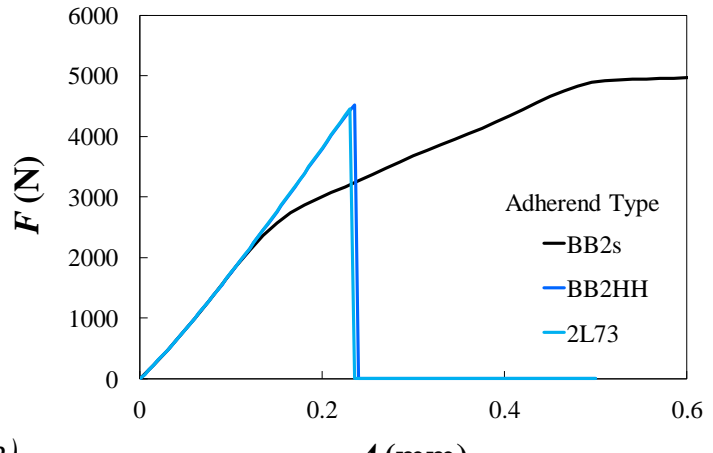

$\Delta(\mathbf{m m})$

Figure 17. (a) Stress-strain relation for bulk adhesive, along with peel and shear components for a single lap joint with $\psi=1.4$, and (b) corresponding load-displacement plots.

Table 3. Experimental and predicted strengths of the single lap joint.

\begin{tabular}{ccc}
\hline Adherend & Experimental Strength $(\mathrm{kN})$ & Predicted Strength $(\mathrm{kN})$ \\
\hline 2L73 & $4.8 \pm 0.57$ & 4.46 \\
BB2hh & $5.0 \pm 0.38$ & 4.52 \\
BB2s & $3.5 \pm 0.32$ & 5.00 \\
\hline
\end{tabular}


The joint with the $2 \mathrm{~L} 73$ adherends failed without the adherends reaching the yield stress, while the BB2hh adherend joint had small amounts of adherend yielding and the BB2s joint was dominated by the effects of adherend yielding. Looking back to the single lap joint example of Section B, the point was made that adherend yielding is not accurately captured by the current formulation of the joint element. As expected, the specimen with no signs of adherend yielding, 2L73, had a predicted strength well within the experimental error. The specimen with slight yielding, BB2hh, had a predicted strength slightly outside of the error range of the experiment. Finally, the BB2s adherend joint, being totally dominated by adherend yielding, had a predicted strength much higher than the experimental value. However, if one again uses the single lap joint of Section B as an example, one could easily imagine that if adherend plasticity were accounted for in a more accurate manner, the predicted peak load would be somewhere around the elbow where the slope first drops, around $3 \mathrm{kN}$. This would bring the prediction much closer to the experimental value. Unfortunately, as predicted in Section B, the joints with more adherend yielding predict

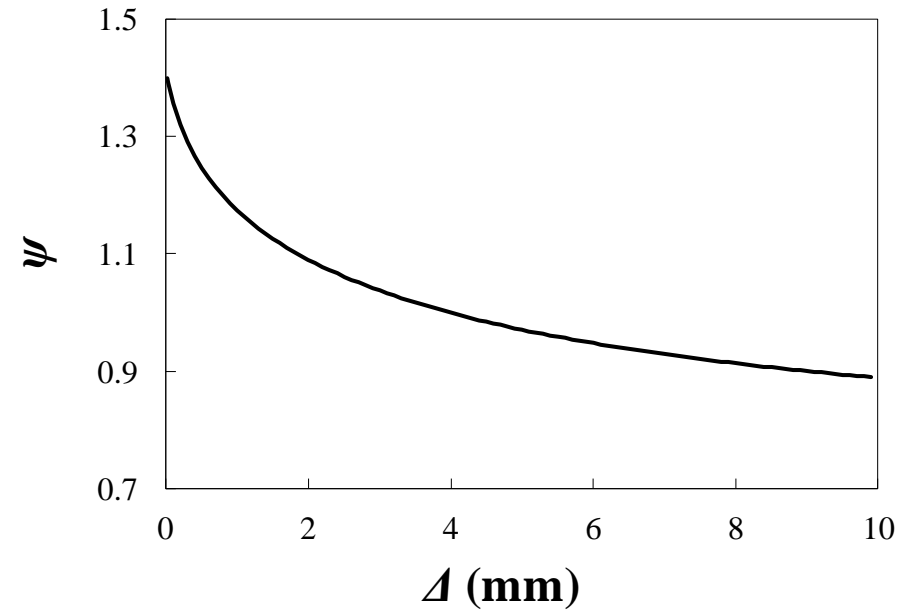

Figure 18. Peel to shear stress ratio in adhesive layer of the single lap joint as a function of end displacement. strengths increasingly deviating from the experimental value.

If the elbow is taken to be the point of failure for the BB2s specimens, all three predictions would be lower than the experimental strength. There are several possibilities for this discrepancy. The first is that the actual joints had quite sizeable fillets at the ends of the adhesive. Although it has been shown that spring-type joint models, like the joint element, predict stresses within the bondline similar to those in joints with fillets ${ }^{5}$, the fillet might reduce the stress enough to increase the strength slightly. Furthermore, the peel to shear ratio, $\psi$, was only approximated base on the linear elastic joint. However, large rotations and the accompanying nonlinearities change the peel to shear ratio, making it a function of the loading. Figure 18 shows the value of $\psi$ as a function of the end displacement, $\Delta$. It can be seen that the peel to shear ratio drops early on in the loading. Therefore, $\psi$ could be adjusted to yield a more accurate answer.

This comparison showed that, as expected, the joint element is less than accurate with regards to adherend material nonlinearity. However the method devised to use bulk adhesive tensile data appears to have been successful in approximating the strength of this single lap joint. For most advanced composite joints, the adherends display brittle failure, so capturing adherend yielding is of secondary importance. However, a more precise model could be implemented to consider adherend damage.

\section{Conclusion}

In this study, the linear elastic joint element concept was extended to include large rotations, material nonlinearity, and adhesive failure. Large rotations, which occur commonly in adhesively bonded joints, were handled through a co-rotational formulation. This formulation separated the displacements into rigid body displacement and local deformations about some rotated local coordinate system. The local deformations are assumed to be small, so a linear formulation can still be used. Material nonlinearities were included into the formulation. However, a nonlinear-elastic model was adopted for simplicity. It was shown through examples that, while this model was sufficient for the adhesive layers with high stress concentrations and often small plastic zones, it was not accurate for a description of the adherend materials, especially in the post-yielded stated. Problems arise with excessive adherend yielding and it is suggested the modeling of such joints with the joint element be avoided. On a positive note, the joint load associated with adherend yielding can be viewed as an upper limit load for the structural joint, predicted using the joint element.

Adhesive failure and crack formation and growth were accounted for through an internal re-meshing process. The element with an internal crack was replaced by a sub-assembly with the failed adhesive removed. This method added to the computational steps that needed to be taken during the analysis, but decreased the number of elements needed to capture progressive failure. 
Finally, methods of finding the nonlinear peel and shear stress-strain curves for the adhesive based on experimental procedures were outlined. First, using bulk adhesive tensile data, the response was broken up into shear and peel components for a certain joint configuration. This allowed the adhesive to be characterized with one test, but limited the shear and peel characterization to be specific to a certain joint type, geometry, and materials. Next, the resemblance of the adhesive model to cohesive zone models made it a natural candidate for fracture properties such as strength and fracture toughness. Tests were conducted to isolate the shear and peel "modes" and characterize them separately. This has the disadvantage of requiring more tests, but seems to have fewer assumptions involved.

\section{Acknowledgments}

Portions of this work were financially supported by the Space Vehicle Technology Institute under grant NCC3989 jointly funded by NASA and the Department of Defense. The buld of the financial support was provided by NASA Glenn Research Center through the GSRP Fellowship.

\section{References}

1. Volkersen O. Die Nietkraftverteilung in Zugbeanspruchten mit Konstanten Laschenquerschritten. Luftfahrtforschung. $1938 ; 15: 41-47$.

2. Goland, Reissner E. The stresses in cemented joints. Journal of Applied Mechanics. 1944;11:A17-A27.

3. Hart-Smith LJ. Adhesive-bonded single-lap joints. NASACR112236. 1973:116.

4. Delale F, Erdogan F, Aydinoglu M n. Stresses in Adhesively Bonded Joints: A Closed-Form Solution. Journal of Composite Materials. 1981;15(3):249-271.

5. Mortensen F, Thomsen OT. Analysis of adhesive bonded joints: a unified approach. Composites Science and Technology. 2002;62(7-8):1011-1031.

6. Gustafson PA, Waas AM. A bonded joint finite element for a symmetric double lap joint subjected to mechanical and thermal loads. International Journal for Numerical Methods in Engineering. 2009;79(1):94-126.

7. Stapleton SE, Waas AM. Reduced-order Modeling Of Adhesively Bonded Joints Using An Enhanced Joint Finite Element. In: Proceedings of the 52nd International SAMPE Symposium. Salt Lake City, UT; 2010. Available at: http://www.sampe.org/store/paper.aspx?pid=6305. Accessed February 9, 2012.

8. Stapleton SE, Waas AM, Arnold SM. Functionally graded adhesives for composite joints. International Journal of Adhesion and Adhesives. 2012;35:36-49.

9. Stapleton SE, Waas A. Macroscopic Finite Element for a Single Lap Joint. In: AIAA/ASME/ASCE/AHS/ASC 50th SDM Conference. Palm Springs, California; 2009.

10. Kafkalidis MS, Thouless MD. The effects of geometry and material properties on the fracture of single lap-shear joints. International Journal of Solids and Structures. 2002;39(17):4367-4383.

11. Harris JA, Adams RA. Strength prediction of bonded single lap joints by non-linear finite element methods. International Journal of Adhesion and Adhesives. 1984;4(2):65-78.

12. Banea MD, da Silva LFM. Adhesively bonded joints in composite materials: An overview. Proceedings of the Institution of Mechanical Engineers, Part L: Journal of Materials Design and Applications. 2009;223(1):1 -18.

13. Belytschko T, Hsieh BJ. Non-linear transient finite element analysis with convected co-ordinates. International Journal for Numerical Methods in Engineering. 1973;7(3):255-271.

14. Crisfield MA, Moita GF. A unified co-rotational framework for solids, shells and beams. International Journal of Solids and Structures. 1996;33(20-22):2969-2992.

15. Crisfield MA. Non-Linear Finite Element Analysis of Solids and Structures. Wiley; 1996. 
16. Guyan RJ. Reduction of stiffness and mass matrices. AIAA Journal. 1965;3(2):380-380.

17. Chen S, Pan HH. Guyan reduction. Communications in Applied Numerical Methods. 1988;4(4):549-556.

18. Chandrupatla TR, Belegundu AD. Introduction to Finite Elements in Engineering. 2 Har/Dsk. Prentice Hall College Div; 1996.

19. Sechler EE. Elasticity in engineering. Dover Publications; 1968.

20. Raghava R, Caddell RM, Yeh GSY. The macroscopic yield behaviour of polymers. Journal of Materials Science. 1973;8(2):225-232.

21. Gali S, Dolev G, Ishai O. An effective stress/strain concept in the mechanical characterization of structural adhesive bonding. International Journal of Adhesion and Adhesives. 1981;1(3):135-140.

22. Li S, Thouless MD, Waas AM, Schroeder JA, Zavattieri PD. Use of a cohesive-zone model to analyze the fracture of a fiberreinforced polymer-matrix composite. Composites Science and Technology. 2005;65(3-4):537-549.

23. Xie D, Waas AM, Shahwan KW, Schroeder JA, Boeman RG. Fracture criterion for kinking cracks in a tri-material adhesively bonded joint under mixed mode loading. Engineering Fracture Mechanics. 2005;72(16):2487-2504.

24. Gustafson PA. Analytical and Experimental Methods for Adhesively Bonded Joints Subjected to High Temperatures. 2008. Available at: http://deepblue.lib.umich.edu/handle/2027.42/60791. Accessed February 9, 2012.

25. Gustafson PA, Waas AM. The influence of adhesive constitutive parameters in cohesive zone finite element models of adhesively bonded joints. International Journal of Solids and Structures. 2009;46(10):2201-2215.

26. Armero F, Ehrlich D. Numerical modeling of softening hinges in thin Euler-Bernoulli beams. Computers \& Structures. 2006;84(10-11):641-656.

27. Armero F, Ehrlich D. An analysis of strain localization and wave propagation in plastic models of beams at failure. Computer Methods in Applied Mechanics and Engineering. 2004;193(30-32):3129-3171.

28. Ehrlich D, Armero F. Finite element methods for the analysis of softening plastic hinges in beams and frames. Computational Mechanics. 2005;35(4):237-264.

29. Vratanar B, Saje M. A consistent equilibrium in a cross-section of an elastic-plastic beam. International Journal of Solids and Structures. 1999;36(2):311-337.

30. Jirásek M. Analytical and Numerical Solutions for Frames with Softening Hinges. Journal of Engineering Mechanics. 1997;123(1):8-14.

31. Wackerfuß J. Efficient finite element formulation for the analysis of localized failure in beam structures. International Journal for Numerical Methods in Engineering. 2008;73(9):1217-1250.

32. Darvall PL, Mendis PA. Elastic-Plastic-Softening Analysis of Plane Frames. Journal of Structural Engineering. 1985;111(4):871-888.

33. Bažant ZP, Pijaudier-Cabot G, Pan J. Ductility, Snapback, Size Effect, and Redistribution in Softening Beams or Frames. Journal of Structural Engineering. 1987;113(12):2348-2364.

34. Crocombe AD. Global yielding as a failure criterion for bonded joints. International Journal of Adhesion and Adhesives. 1989;9(3):145-153. 